\title{
Resveratrol attenuates intermittent hypoxia-induced insulin resistance in rats: Involvement of Sirtuin 1 and the phosphatidylinositol-4,5-bisphosphate 3-kinase/AKT pathway
}

\author{
QIUYAN WANG ${ }^{1}$, XIAOHUI SUN ${ }^{2}$, XIAOBIN LI $^{2}$, XIANG DONG $^{2}$, PENG LI $^{1}$ and LI ZHAO ${ }^{1}$ \\ ${ }^{1}$ Department of Respiratory Medicine, Shengjing Hospital of China Medical University, Shenyang, Liaoning 110001; \\ ${ }^{2}$ Emergency Department, First Affiliated Hospital of Dalian Medical University, Dalian 116011, P.R. China
}

Received February 9, 2014; Accepted October 1, 2014

DOI: $10.3892 / \mathrm{mmr} .2014 .2762$

\begin{abstract}
Obstructive sleep apnea can induce chronic intermittent hypoxia (CIH) during sleep and is associated with obesity and diabetes. Resveratrol (RSV), a polyphenolic phytoalexin, can regulate glucose metabolism, thereby reducing insulin resistance. The present study aimed to assess whether RSV attenuates $\mathrm{CIH}$-induced insulin resistance in rats and the underlying mechanisms. A total of 40 rats were randomly assigned into five groups: i) Control; ii) subjected to CIH only; iii) subjected to CIH and treated with $3 \mathrm{mg} / \mathrm{kg} / \mathrm{day}$ of RSV; iv) subjected to CIH and treated with $30 \mathrm{mg} / \mathrm{kg} / \mathrm{day}$ of RSV; v) subjected to CIH and treated with $60 \mathrm{mg} / \mathrm{kg} / \mathrm{day}$ of RSV All animals were sacrificed following 28 days of treatment. Subsequently, the blood and livers were harvested and blood insulin and glucose levels were measured. Levels of sirtuin (Sirt) 1, insulin receptor (InsR) and glucose transporter 2 (Glut2) in the liver were measured. RSV treatment was demonstrated to suppress weight gain and improve hepatic morphology. RSV treatment also significantly reduced the homeostasis model assessment estimate of insulin resistance of the rats exposed to $\mathrm{CIH}$. This effect occurred in a dose-dependent manner. RSV significantly upregulated liver Sirtl levels and inhibited InsR and Glut2 expression in the liver. Additionally, RSV activated the phosphorylation of phosphatidylinositol-4,5-bisphosphate 3-kinase (PI3K) and AKT. The present study demonstrates that RSV prevents $\mathrm{CIH}$-induced insulin resistance in rats. Upregulation of Sirt1 and activation of PI3K/AKT signaling may be involved in this process.
\end{abstract}

Correspondence to: Dr Li Zhao, Department of Respiratory Medicine, Shengjing Hospital of China Medical University, 36 Sanhao Street, Heping, Shenyang, Liaoning 110001, P.R. China E-mail: 1zhaoli@163.com

Key words: resveratrol, intermittent hypoxia, insulin resistance, sirtuin 1 , obstructive sleep apnea

\section{Introduction}

Obstructive sleep apnea (OSA) is a complex pathophysiological condition affecting 1-4\% of the general population and $25-35 \%$ of obese individuals $(1,2)$. It is characterized by repetitive upper airway obstruction, frequent snoring, apnea during sleep, chronic sleep loss at night and excessive daytime sleeping (3). OSA can induce chronic intermittent hypoxia $(\mathrm{CIH})$ during sleep. There are established associations with obesity, insulin resistance (IR), glucose intolerance and other metabolic syndromes (4). Clinical and epidemiological studies have suggested that sleep disturbances are associated with a group of metabolic disorders, including IR, diabetes mellitus, hypertension and dyslipidemia (5-9). In particular, sleep loss is an independent risk factor affecting the development and progression of diabetes. Sleep loss contributes to IR and diabetes either by directly interfering with the regulation of glucose metabolism, or indirectly through weight gain and obesity by affecting appetite (10).

Resveratrol (trans-3,5,4-trihydroxystilbene, RSV), a natural polyphenolic phytoalexin, is highly concentrated in grapes and red wine. RSV is known to promote weight loss, reduce IR and improve disease symptoms in patients with diabetes $(11,12)$. RSV has been hypothesized to be a potent activator of the mammalian silent information regulator gene Sirtuin 1 (SIRT1) $(12,13)$. Previously, SIRT3 and SIRT5 were identified as direct targets of RSV (14).

Sirtuins are a group of nicotinamide adenine dinucleotide-dependent protein deacetylases (15). There are seven members in this family, including Sirt 1-7. Sirtuins are involved in numerous biological functions involving cell growth, apoptosis, energy metabolism and the stress response (16-18). Sirt1 is widely expressed in mammalian tissues. Activation of Sirt1 promotes insulin secretion, reduces glucose tolerance and decreases body weight $(19,20)$. Animal studies have demonstrated that SIRTI deficiency in liver leads to hyperglycemia, oxidative damage and insulin resistance (21). Sirtl is important in regulating energy metabolism and glucose homeostasis. Blood glucose levels are tightly regulated within a narrow range and hypoglycemia and hyperglycemia have a detrimental effect. Glucose homeostasis is critically dependent on the levels of insulin released by pancreatic $\beta$-cells and on the ability of insulin to inhibit the production of hepatic glucose, while promoting 
the uptake of glucose in peripheral tissues. The responsiveness of the $\beta$-cells as well as insulin sensitivity are affected by sleep $(22,23)$. Sleep loss and IR are closely associated with OSA. RSV has been reported to reduce IR and maintain blood glucose levels within the normal range. However, there are few studies on the efficacy of RSV in treating patients with OSA. In order to investigate the potential therapeutic application of RSV for treating patients with OSA, a chronic intermittent hypoxic model in rats was generated to mimic the chronic hypoxia and IR presented in patients with OSA. The aim of the present study was to determine the efficacy of RSV in reducing $\mathrm{CIH}$-induced IR in rats and the underlying molecular mechanisms.

\section{Materials and methods}

Animals. A total of 40 eight-week-old male Sprague-Dawley rats purchased from Chang Sheng Biotechnology Corp. (Liaoning, China) were used in the present study. Animals were housed in the animal facility at the Shenghai Hospital of China Medical University (Shenyang, China) with standard $12 \mathrm{~h}$ light/dark cycles and ad libitum access to food and water. All animal procedures were approved by the Institutional Animal Care and Use Committee of the Shengjing Hospital of China Medical University (Shenyang, China). RSV was purchased from Guanyu Biotechnology Corp. (Shanxi, China). Animals were randomly assigned into five different groups with eight rats in each group: i) Control group: animals were exposed to normal conditions without RSV treatment; ii) CIH group: animals were exposed to intermittent hypoxic conditions without RSV treatment; iii) CIH plus low RSV group: animals were exposed to intermittent hypoxic conditions and administered daily with $3 \mathrm{mg} / \mathrm{kg}$ /day of RSV; iv) CIH plus median RSV group: animals were exposed to intermittent hypoxic conditions and administered daily with $30 \mathrm{mg} / \mathrm{kg} /$ day of RSV; v) CIH plus high RSV group: animals were exposed to intermittent hypoxic conditions and administered daily with $60 \mathrm{mg} / \mathrm{kg}$ /day of RSV. RSV was prepared by adding $3 \mathrm{ml}$ water. Rats were fed via oral gavage with $3 \mathrm{ml} \mathrm{RSV}$ daily, for a total of 28 days. Rats in control groups without RSV treatment were administered $3 \mathrm{ml}$ of water as vehicle via an oral gavage. The body weight of animals was recorded every week for all animals. Blood glucose levels and insulin levels were recorded at the end of the experiment for all animals.

CIH exposure. Animals were exposed to intermittent hypoxia every day for 8 h, from 9 a.m. to 5 p.m., for 28 days, as described previously $(24,25)$. Briefly, housing cages were placed inside plexiglas chambers where the oxygen level was monitored and regulated by timer-controlled valves connected to room air source and a nitrogen source. The air and nitrogen entered the chamber via separate flow meters. During this period of time, oxygen was reduced from $21 \%$ to $10 \%$ over 1.5 min, maintained at $10 \%$ for $1.5 \mathrm{~min}$, returned to $21 \%$ over $1 \mathrm{~min}$ and held at $21 \%$ for $2 \mathrm{~min}$. This cycle was repeated for $8 \mathrm{~h}$. Control animals were housed in identical chambers for an equivalent amount of time and were exposed to the same timer and valve-controlled changes in air flow as the CIH rats; however, the only source of gas in the control chambers was room air, so they remained at normoxic levels throughout the investigation. Immediately following the final hypoxia session, the cages were returned to the main housing room. The animals were fasted for $12 \mathrm{~h}$ prior to day 29 following the treatment and then sacrificed under isoflurane anesthesia. Liver tissues and blood samples were collected.

Histology. Animals were sacrificed in a preprandial state under isoflurane anesthesia on day 29. Liver tissues were collected from all animals. All tissues were rapidly frozen in liquid nitrogen and stored at $-80^{\circ} \mathrm{C}$ for future investigation. Paraffin-embedded tissue sections ( $4 \mu \mathrm{m}$ thick) were obtained and were stained with hematoxylin and eosin (H\&E).

Detection of blood glucose and insulin levels. Blood glucose concentration was measured using a Contour glucometer (Bayer, Pittsburgh, PA, USA) and Contour blood glucose test strips (Bayer). Plasma insulin was determined using a Rat Insulin ELISA kit (Millipore, Billerica, MA, USA) according to the manufacturer's instructions. The value of the homeostatic model assessment (HOMA)-IR index was calculated using the following formula: [Fasting insulin concentration $(\mu \mathrm{U} / \mathrm{ml}) \times$ fasting glucose concentration $(\mathrm{mmol} / \mathrm{l})] / 22.5$.

Immunohistochemistry $(I H C)$. Sirtl protein expression in paraffin-embedded hepatic tissue sections was detected using a standard avidin-biotin immunoperoxidase protocol. Briefly, liver sections were deparaffinized and pretreated with $1 \% \mathrm{H}_{2} \mathrm{O}_{2}$ for $30 \mathrm{~min}$ to quench endogenous peroxidase activity, followed by three rinses in phosphate-buffered saline (PBS). The samples were blocked with $10 \%$ goat serum (Solarbio, Beijing, China) and subsequently incubated with 1:50 dilution of polyclonal rabbit anti-rat Sirt1 antibody (Santa Cruz Biotechnology, Inc., Santa Cruz, CA, USA) overnight at $4^{\circ} \mathrm{C}$. This was followed by incubation with 1:500 dilution of polyclonal goat anti-rabbit secondary antibody (Beyotime, Shanghai, China) at room temperature for $1 \mathrm{~h}$. Immune complexes were detected using an avidin-3,3'-diaminobenzidine staining kit (Beijing Zhongshan Biotechnology, Beijing, China). The sections were counterstained with hematoxylin and mounted. Images of the slides were captured using a stereo BX51 light microscope (Olympus, Tokyo, Japan) equipped with a digital camera.

$R N A$ analysis and reverse transcription quantitative polymerase chain reaction ( $R T-q P C R)$. SIRT1 expression was determined by RT-qPCR as described previously (26). Briefly, total RNA was isolated from rat liver using TRIzol reagent (Invitrogen Life Technologies, Carlsbad, CA, USA) according to the manufacturer's instructions. cDNA was synthesized by RT using the QuantiTec Reverse Transcription kit (Tiangen Biotech, Beijing, China). Subsequently, qPCR was performed using the following primers: Sirtl, forward 5'-ACCCTCAATTTCTGTTCTGC-3' and reverse 5'-TTGGACATTACCACGTCTGC-3'; $\beta$-actin, forward 5'-GGAGATTACTGCCCTGGCTCCTAGC-3' and reverse 5'-GGCCGGACTCATCGTACTCCTGCTT-3'. The cycle threshold $(\mathrm{Ct})$ values of the target gene were first normalized to $\beta$-actin from the same sample and subsequently the relative differences between the groups were calculated and expressed as relative mRNA levels, setting the control group as 1. Each sample was assessed in triplicate. 
Western blotting. Liver tissue was homogenized in radio immunoprecipitation lysis buffer (Beyotime) supplemented with protease inhibitors (PMSF and aprotonin) and protein phosphatase inhibitor ( $\mathrm{NaF}$ and sodium orthovandate). The protein concentration was determined using a bicinchoninic acid assay protein assay kit (Beyotime). Protein aliquots $(40 \mu \mathrm{g})$ were separated on a $10 \%$ sodium dodecyl sulfate polyacrylamide gel electrophoresis gel and transferred onto a polyvinylidene difluoride membrane. The membrane was blocked with $5 \%$ non-fat milk in PBS and incubated overnight at $4^{\circ} \mathrm{C}$ with one of the following primary antibodies: Rabbit polyclonal anti-SIRT1 (1:400; Santa Cruz Biotechnology, Inc.); rat polyclonal anti-insulin receptor (InsR; 1:1,000; Wuhan Boster Biological Technology, Ltd., Wuhan, China); rabbit polyclonal anti-glucose transporter 2 (Glut2, 1:400; Santa Cruz Biotechnology, Inc.); rabbit polyclonal anti-phospho-Tyr467-phosphatidylinositol-4,5-bisphosphate 3-kinase (PI3K; 1:400; Santa Cruz Biotechnology, Inc.); rabbit polyclonal anti-total-PI3K (1:1,000; Wuhan Boster Biological Technology, Ltd.); rabbit polyclonal anti-phospho-Ser473-AKT (1:400; Santa Cruz Biotechnology, Inc.); rabbit polyclonal anti-total-AKT (1:400; Wuhan Boster Biological Technology, Ltd.). Following incubation with the primary antibody, the membranes were washed and incubated with horseradish peroxidase-conjugated secondary antibodies at $37^{\circ} \mathrm{C}$ for $45 \mathrm{~min}$. Enhanced chemiluminescence reagent (Millipore) was used for detection of protein bands. The densities of the protein bands were determined using a gel imaging system (Beijing Sixty-One Instrument, Beijing Six-One Instrument, Beijing, China). The expression levels of Sirt1, InsR, total PI3K, phosphorylated-PI3K, total AKT, phosphorylated-AKT and Glut2 protein levels were normalized against those of $\beta$-actin levels.

Statistical analysis. Data are presented as the mean \pm standard deviation. For a comparison between two groups, Student's t-test was used. For comparisons among three or more groups, one-way analysis of variance was used. $\mathrm{P}<0.05$ was considered to indicate a statistically significant difference.

\section{Results}

$R S V$ suppresses $C I H$-induced body weight gain in rats. Weight gain is associated with chronic hypoxia in animals and humans. To evaluate the effect of RSV on body weight in animals following hypoxia exposure, the weight of each animal was recorded every week. Prior to exposure to hypoxia, all rats had similar body weights. Compared with the control group, the body weight in rats significantly increased following $\mathrm{CIH}$ exposure. At day 29, the average body weight of control rats was $297 \mathrm{~g}$ and that of $\mathrm{CIH}$-treated rats was $388 \mathrm{~g}(\mathrm{P}=0.000)$. The body weights in rats treated with RSV decreased; the average weight was $372.125 \mathrm{~g}$ for the CIH plus low RSV group, $323.675 \mathrm{~g}$ for the CIH plus median RSV group and $370.250 \mathrm{~g}$ for the $\mathrm{CIH}$ plus high RSV group. Compared with the $\mathrm{CIH}$ group, there were significant differences among RSV-treated groups $(\mathrm{P}<0.01)$. These results demonstrate that $\mathrm{CIH}$ increases the body weight of rats, however, RSV inhibits this weight gain (Fig. 1).

$R S V$ reverses $C I H$-induced $I R$ in rats. To evaluate the effect of RSV on IR following chronic hypoxia exposure, the blood glucose and insulin levels of animals was measured on

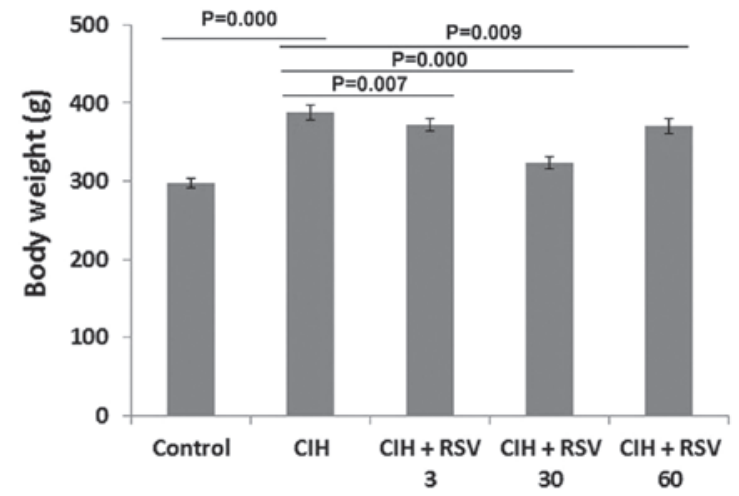

Figure 1 . Body weights of rats. Rats were treated daily with or without RSV $(3,30$ or $60 \mathrm{mg} / \mathrm{kg} /$ day $)$ via an oral gavage. Rats were placed daily in intermittent hypoxic chambers for $8 \mathrm{~h}$ each day for 28 days. Control rats were placed in normoxic chambers. On day 29, following the treatment, the body weights of rats ( $n=8$ in each group) were recorded. Compared with the control group, the body weights of rats significantly increased in the $\mathrm{CIH}$-treated groups $(\mathrm{P}=0.000)$. Compared with the $\mathrm{CIH}$ group, the body weights of rats significantly decreased in the CIH-treated groups following RSV treatment (all $\mathrm{P}<0.001$ ). RSV, resveratrol; $\mathrm{CIH}$, chronic intermittent hypoxia.

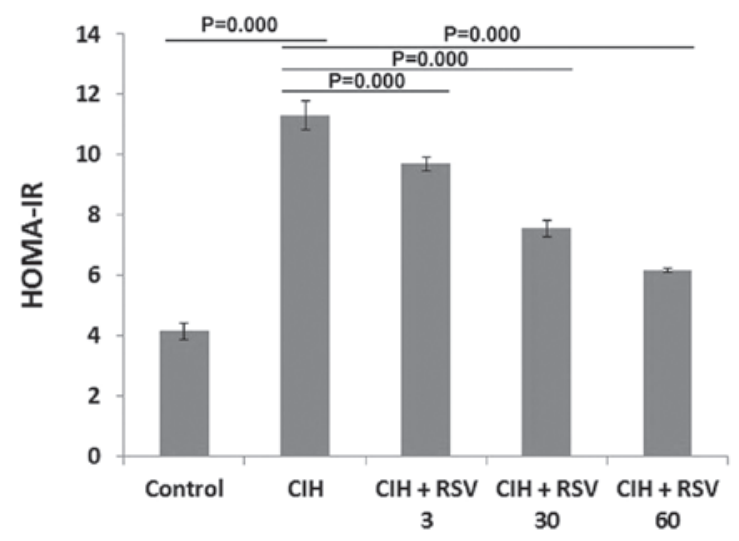

Figure 2. HOMA-IR index in rats. Rats were treated daily with or without $\operatorname{RSV}(3,30$ or $60 \mathrm{mg} / \mathrm{kg} /$ day) via an oral gavage. Rats were placed daily in intermittent hypoxic chambers for $8 \mathrm{~h}$ each day for 28 days. Control rats were placed in normoxic chambers. On day 29 following the treatment, blood was collected from all animals. Blood glucose levels were measured using a Contour glucometer. Blood insulin levels were measured using a rat insulin ELISA kit. The HOMA-IR index was calculated using the following formula: [Fasting insulin concentration $(\mu \mathrm{U} / \mathrm{ml}) \mathrm{x}$ fasting glucose concentration $(\mathrm{mmol} / \mathrm{l})] / 22.5$, assuming that normal subjects have an insulin resistance of 1 . CIH increased the HOMA-IR in rats ( $\mathrm{n}=8$ for each group) and RSV significantly reversed this effect. The HOMA-IR index significantly increased in the CIH-exposed group compared with the control group $(\mathrm{P}=0.000)$ and significantly decreased in RSV-treated groups $(\mathrm{P}=0.000)$. RSV, resveratrol; $\mathrm{CIH}$, chronic intermittent hypoxia; HOMA-IR, homeostasis model assessment-estimated insulin resistance.

day 29 following hypoxia exposure. IR was assessed using the HOMA-IR index. Compared with the control group, the HOMA-IR values of CIH-treated groups were significantly higher $(\mathrm{P}=0.000)$. The values were $4.14 \pm 0.29$ (control group), $11.32 \pm 0.47$ (CIH group), 9.70 \pm 0.24 (CIH plus low RSV group), $7.55 \pm 0.29$ (CIH plus median RSV group) and 6.16 $\pm 0.06(\mathrm{CIH}$ plus high RSV group), respectively. Compared with the $\mathrm{CIH}$ without RSV group, the values significantly decreased in other CIH-treated groups following RSV treatment $(\mathrm{P}=0.000)$. The reduction in RSV-treated groups was dose dependent. 


\section{Control}

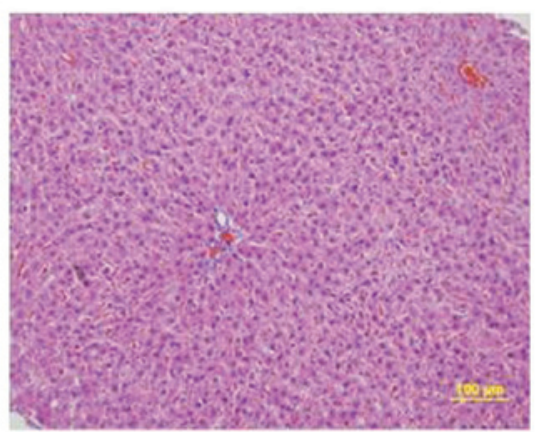

$\mathrm{CIH}$

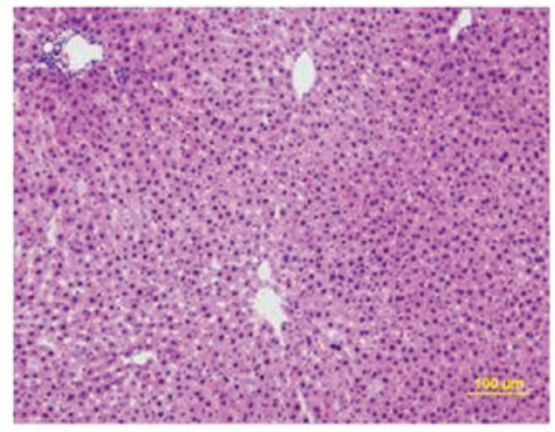

\section{CIH + RSV 3}

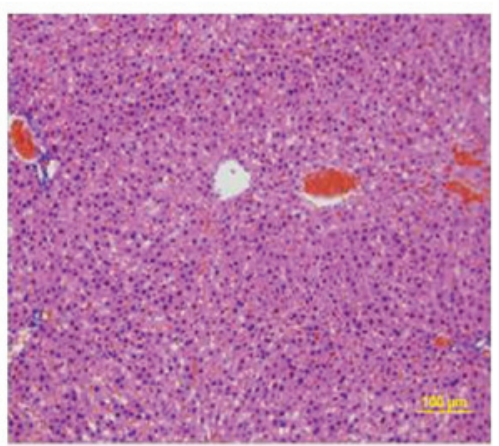

CIH + RSV 30

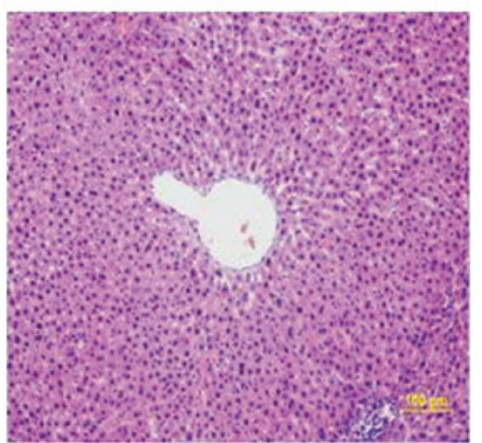

CIH + RSV 60

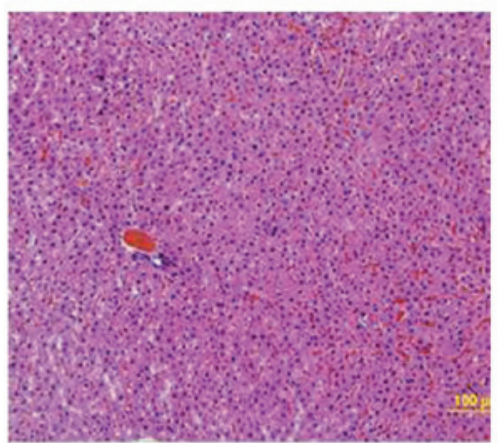

Figure 3. Morphology of liver tissue in rats. Rats were treated daily with or without RSV (3, 30 or $60 \mathrm{mg} / \mathrm{kg} / \mathrm{day}) \mathrm{via}$ an oral gavage. Rats were placed daily in intermittent hypoxic chambers for $8 \mathrm{~h}$ each day for 28 days. Control rats were placed in normoxic chambers. On day 29 following the treatment, animals were sacrificed in a postprandial state under isoflurane anesthesia. Livers were collected for histological analysis. Liver tissue was routinely fixed and embedded in paraffin blocks. Paraffin-embedded tissues ( $4 \mu \mathrm{m}$ thick) were sectioned and stained with hematoxylin and eosin (magnification, $\mathrm{x} 100)$. RSV, resveratrol; CIH, chronic intermittent hypoxia.

However, the values in CIH-exposed groups remained higher than that observed in the control group regardless of RSV treatment (Fig. 2).

$R S V$ protects rats against $\mathrm{CIH}$-induced liver damage. To evaluate the morphological alterations in the rat liver following $\mathrm{CIH}$ exposure and the effect of RSV on these changes, liver tissue sections were stained with H\&E. The hepatocytes in the control rat tissue were tightly arranged together with clearly visible nuclei. The hepatocytes in the $\mathrm{CIH}$-treated rats were swollen accompanied with nuclear pyknosis, degeneration and only partial cytoplasmic vacuoles were present. The changes in the group treated with a low dose of RSV were similar to that observed in the CIH without RSV group, for example, swollen hepatocytes, fuzzy cell boundaries, nuclear pyknosis and the presence of partial cytoplasmic vacuole were observed. The cell boundaries in the median RSV treated rats were less fuzzy than those observed in the control group. The changes in the group treated with a high dose of RSV appeared normal. These observations suggested that RSV may protect the rat against CIH-induced liver damage (Fig. 3).

$R S V$ upregulates the mRNA and protein levels of Sirtl in the liver. RSV is hypothesized to be the most potent activator of Sirt1. To evaluate the effect of RSV on Sirt1 levels in liver following $\mathrm{CIH}$ exposure, mRNA and protein levels of Sirt1 in liver tissue were analyzed. Compared with the control group, Sirt1 protein levels in CIH-treated groups significantly reduced following $\mathrm{CIH}$ exposure $(\mathrm{P}=0.000)$. However, the levels in the RSV-treated $\mathrm{CIH}$ groups were elevated compared with that in the CIH group without RSV treatment $(\mathrm{P}=0.000)$. These changes were dose dependent (Fig. 4A). The mRNA changes of SIRTI were similar to that observed in the protein levels. SIRT1 mRNA levels in the hepatic tissue were significantly reduced following $\mathrm{CIH}(\mathrm{P}=0.000)$, however, RSV treatment significantly increased the mRNA levels $(\mathrm{P}=0.000$; Fig. 4B). The IHC staining revealed a significant reduction in the SIRT1-positive cells in hepatic tissue sections from rats subjected to $\mathrm{CIH}$ compared with that detected in the control group, however, RSV treatment led to an increase in Sirt1-positive cells (Fig. 4C). These results suggested that RSV significantly upregulated the levels of Sirt1 in rats following $\mathrm{CIH}$ exposure.

$R S V$ downregulates InsR and Glut2 levels in the liver. InsR and Glut 2 are important in regulating glucose metabolism. The levels are positively correlated with IR and diabetes. To evaluate the effect of RSV on these levels in the liver, the levels of the two proteins were analyzed using western blotting. The gel densities were quantitated and expressed as fold-changes following normalizing against the levels of $\beta$-actin. The relative protein InsR level was significantly higher in the $\mathrm{CIH}$ group compared with that in the control group ( $\mathrm{P}=0.000$; Fig. 5A). Compared with the $\mathrm{CIH}$ without RSV group, RSV treatment significantly reduced InsR levels $(\mathrm{P}=0.042,3 \mathrm{mg} / \mathrm{kg} / \mathrm{day} \mathrm{RSV} ; \mathrm{P}=0.000,30 \mathrm{mg} / \mathrm{kg} /$ day 
A

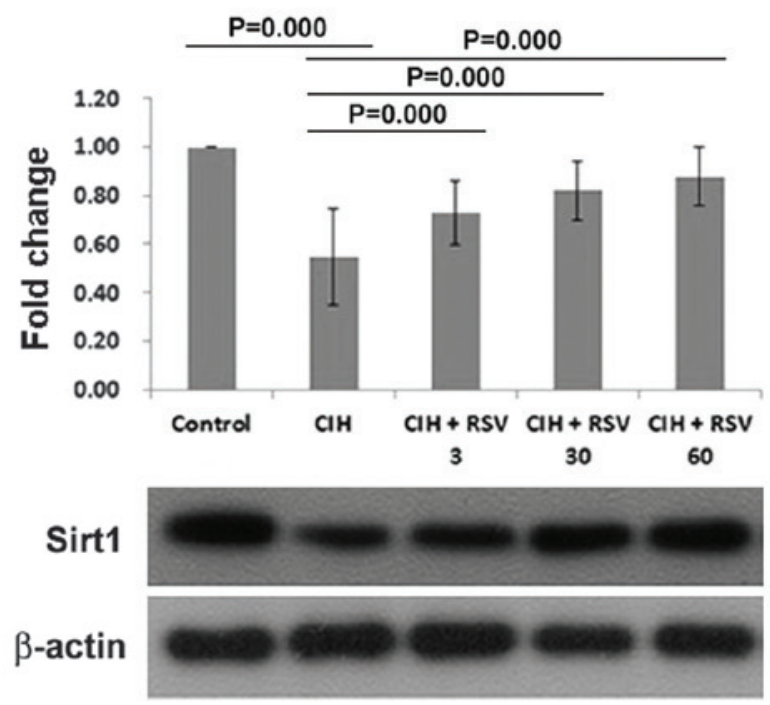

C
B

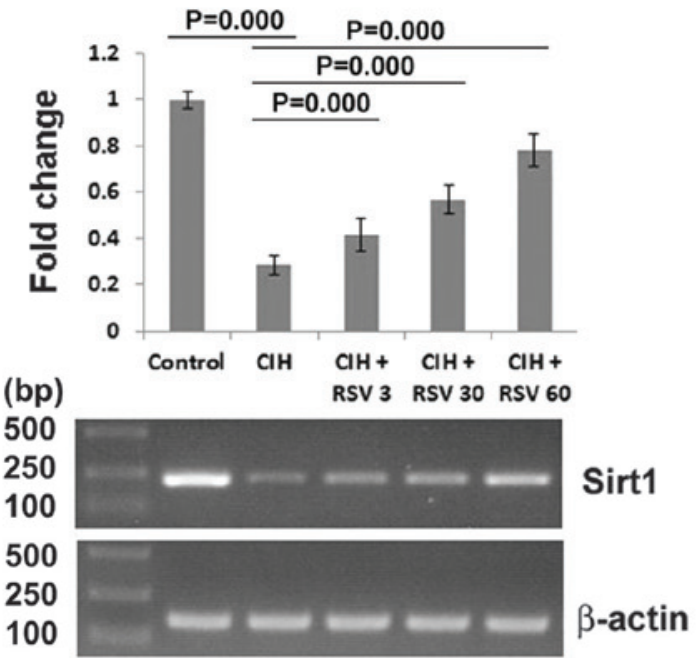

CIH
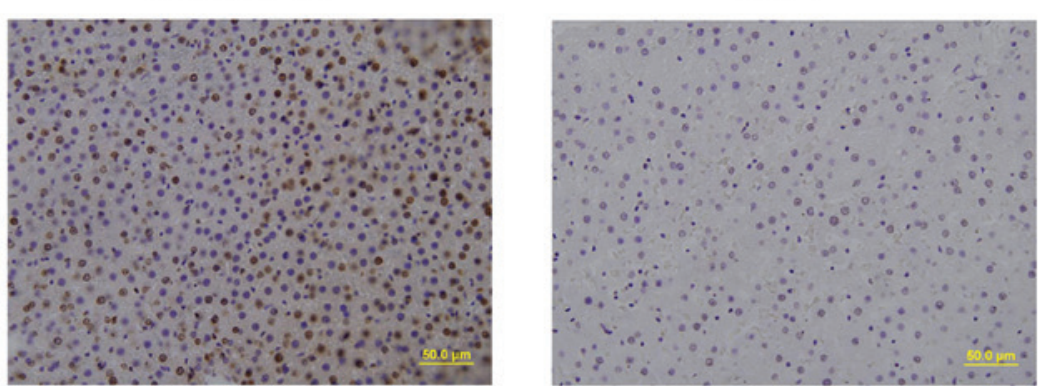

CIH + RSV 3

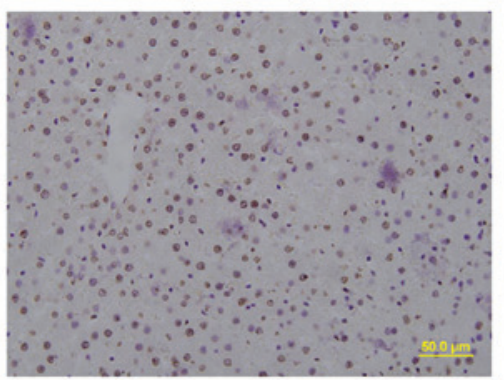

CIH + RSV 30

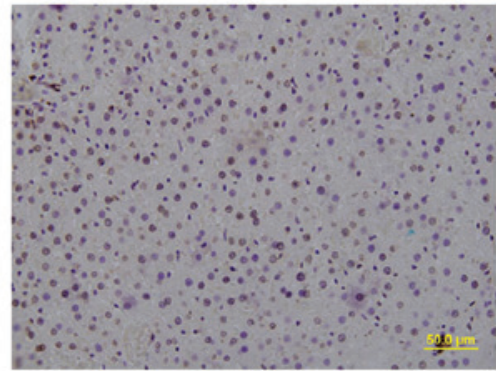

CIH + RSV 60

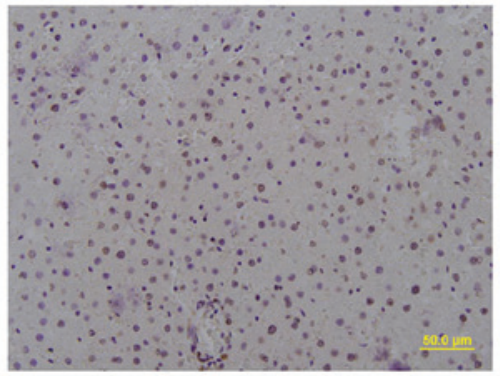

Figure 4. Sirt1 levels in rat liver. Rats were treated daily with or without RSV (3,30 or $60 \mathrm{mg} / \mathrm{kg} /$ day $)$ via an oral gavage. Rats were placed daily in intermittent hypoxic chambers for $8 \mathrm{~h}$ each day for 28 days. Control rats were placed in normoxic chambers. On day 29 following the treatment, rat liver was harvested. (A) Proteins from hepatic tissue were extracted, resolved on a 10\% SDS-PAGE gel, transferred onto a membrane and stained with antibody against Sirt1. (B) Relative mRNA levels of SIRTI in the liver were measured by quantitative polymerase chain reaction. $\beta$-actin was used as an internal control. Sirt1 protein and mRNA measurements were normalized to levels of $\beta$-actin protein and mRNA, respectively, and expressed as fold-change. (C) Paraffin-embedded hepatic tissue sections were stained with antibody against Sirt1 for immunohistochemistry analysis as described in Materials and methods. mRNA and protein levels of Sirt1 significantly decreased in the CIH group compared with that in the control group ( $\mathrm{P}=0.000)$. It significantly increased in RSV-treated groups (all $\mathrm{P}=0.000$ ). RSV, resveratrol; $\mathrm{CIH}$, chronic intermittent hypoxia.

RSV; P=0.000, $60 \mathrm{mg} / \mathrm{kg} / \mathrm{day}$ RSV). The level of InsR in the group treated with a low dose of RSV was higher than that in the normal control, while, the level of InsR in the groups treated with a median and high dose of RSV were similar to that in the normal control (Fig. 5A). The alterations in Glut2 levels were similar to those of InsR. Glut2 levels significantly increased in the $\mathrm{CIH}$ group compared with that in the control group $(\mathrm{P}=0.000)$. Compared with the $\mathrm{CIH}$ without $\mathrm{RSV}$ group, RSV treatments significantly reduced Glut2 levels at 30 and $60 \mathrm{mg} / \mathrm{kg} /$ day of RSV, but not $3 \mathrm{mg} / \mathrm{kg} / \mathrm{day}$ of RSV ( $\mathrm{P}=0.422$,
$3 \mathrm{mg} / \mathrm{kg} /$ day RSV; $\mathrm{P}=0.004,30 \mathrm{mg} / \mathrm{kg} /$ day RSV; $\mathrm{P}=0.000$, $60 \mathrm{mg} / \mathrm{kg} /$ day RSV; Fig. 5B).

RSV activates phosphorylation of PI3K and AKT in the liver. To further investigate the underlying mechanisms by which RSV reduces IR in chronic hypoxic animals, the phosphorylation of PI3K and AKT was analyzed. The data demonstrated that $\mathrm{CIH}$ exposure significantly inhibited the phosphorylation of PI3K and AKT $(\mathrm{P}=0.000)$. RSV treatment significantly upregulated the phosphorylation of PI3K and AKT $(\mathrm{P}=0.000)$. 
A

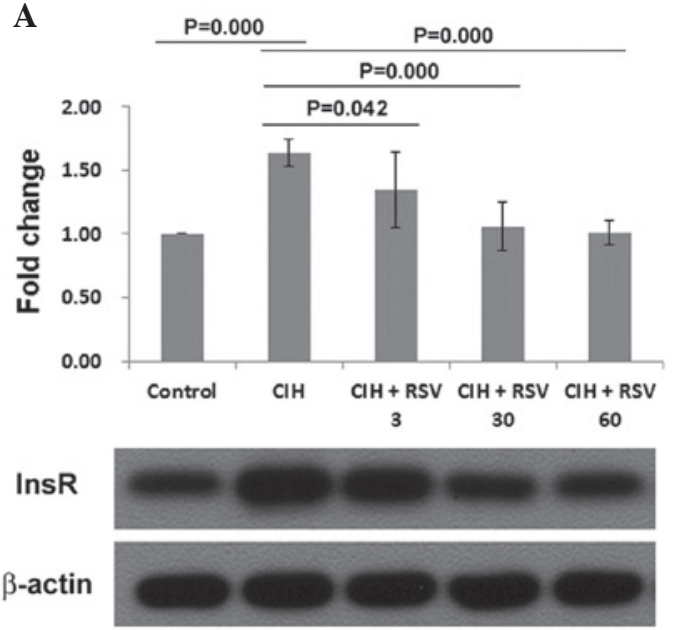

B

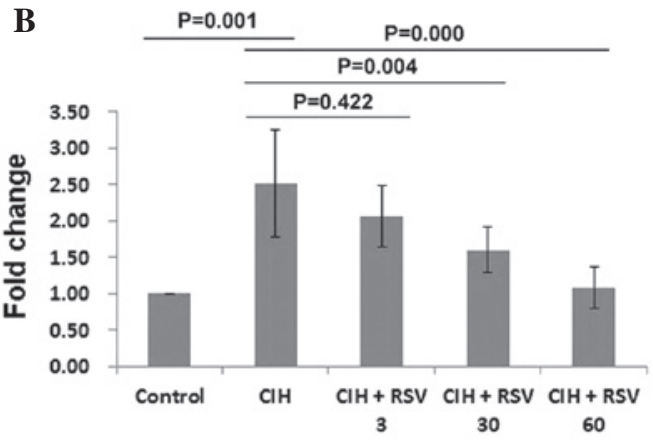

Glut2

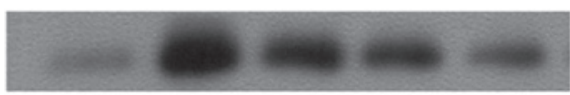

$\beta$-actin

Figure 5. Liver protein levels of InsR and Glut2 in rats. Rats were treated daily with or without RSV (3, $30 \mathrm{or} 60 \mathrm{mg} / \mathrm{kg} / \mathrm{day})$ via an oral gavage. Rats were placed daily in intermittent hypoxic chambers for $8 \mathrm{~h}$ each day for 28 days. Control rats were placed in normoxic chambers. On day 29 following the treatment, rats were sacrificed and liver was harvested. Proteins from hepatic tissue were harvested, resolved on a 10\% SDS-PAGE gel and immunostained with antibody against (A) InsR and (B) Glut2. InsR and Glut2 levels were normalized to levels of $\beta$-actin protein and were expressed as fold change. InsR and Glut2 protein levels significantly increased in the CIH group compared with the control group. The levels decreased in RSV-treated groups. RSV, resveratrol; CIH, chronic intermittent hypoxia; InsR, insulin receptor; Glut2, glucose transporter 2.
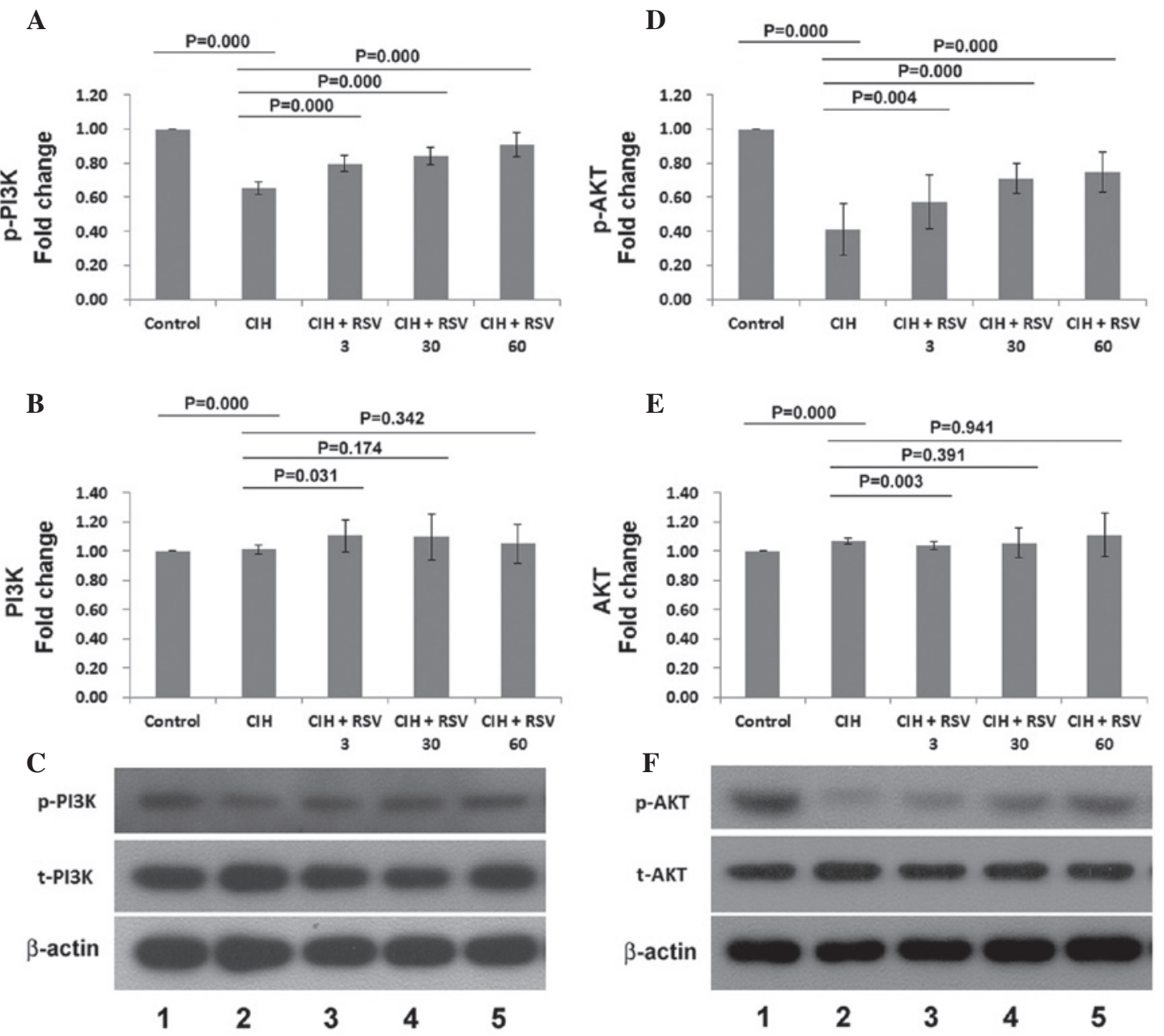

Figure 6. Phosphorylation of PI3K and AKT in rat liver. Rats were treated daily with or without RSV (3,30 or $60 \mathrm{mg} / \mathrm{kg} / \mathrm{day})$ via an oral gavage. Rats were placed daily in intermittent hypoxic chambers for $8 \mathrm{~h}$ each day for 28 days. Control rats were placed in normoxic chambers. On day 29 following the treatment rats were sacrificed and hepatic tissue was harvested. Proteins from hepatic tissue were extracted, resolved on a 10\% SDS-PAGE gel, transferred onto a membrane and stained with antibodies against (A and C) phospho-Tyr467-PI3K, (B and C) t-PI3K and (D and F) phospho-Ser473-AKT and (E and F) t-AKT. Protein measurements were normalized to levels of $\beta$-actin protein in each group and were expressed as fold change. p-PI3K, phosphorylated-PI3K; t-PI3K, total PI3K; p-AKT, phosphorylated-AKT; t-AKT, total AKT; RSV, resveratrol; CIH, chronic intermittent hypoxia; PI3K, phosphatidylinositol-4,5-bisphosphate 3-kinase. 
RSV treatment had no effect on total PI3K and total AKT protein levels following $\mathrm{CIH}$ exposure $(\mathrm{P}>0.05)$ with the exception of $3 \mathrm{mg} / \mathrm{kg} / \mathrm{day} \mathrm{RSV}(\mathrm{P}<0.05)$. Compared with the control group, the total levels of PI3K and AKT in the $\mathrm{CIH}$ group increased ( $\mathrm{P}=0.000$; Fig. 6).

\section{Discussion}

Sleep apnea is considered a major cause of obesity and diabetes in patients suffering from this disorder. Patients with OSA undergo repeated cycles of intermittent hypoxia during sleep. In the present study, rats were exposed to a chronic intermittent hypoxic environment to mimic the episodic hypoxia occurring in patients with OSA. The major findings included: i) $\mathrm{CIH}$ increased the body weights of animals and caused liver damage; ii) CIH induced IR in animals; iii) RSV reversed IR and improved the structure of the liver; iv) Upregulation of Sirt1 and activation of PI3K/AKT signaling are possibly involved in this process.

Chronic hypoxia is often associated with weight gain and obesity. The present study demonstrated that the body weights in CIH-treated animals significantly increased. RSV was able to prevent the weight gain in rats. This effect varied under different doses of RSV. Low and high doses of RSV partially prevented the weight gain, however, a medium dose of RSV significantly prevented the weight gain. The reasons for this difference remain to be elucidated. In addition to preventing body weight gain, RSV can protect rats against $\mathrm{CIH}$-induced liver damage. The high dose of RSV offered optimal protection against hepatic damage.

Episodic hypoxemia poses a severe threat to energy metabolism and glucose homeostasis. In the present study, the HOMA-IR index, which reflected IR, increased significantly in animals exposed to $\mathrm{CIH}$. This suggests that $\mathrm{CIH}$ induces significant IR, which leads to decreased blood insulin levels and increased blood glucose levels. RSV treatment significantly reversed IR induced by CIH in rats. This effect was dose dependent. It was also noted that $\mathrm{CIH}$ significantly increased InsR and Glut2 protein levels in rat hepatic tissues. The levels were significantly reduced with RSV treatment in a dose-dependent manner. These results also suggest that RSV was effective in ameliorating IR induced by CIH and also enhanced insulin sensitivity in vivo. The present results are consistent with previous studies (27-32).

RSV has been suggested as a potent activator of Sirt1. The effects of RSV on metabolism, cancer, aging and inflammation were revealed through upregulation of Sirt1 $(12,34)$. Sirt1 is important in these biological processes (35-37). The importance of Sirt1 activation in energy metabolism has been confirmed in SIRTI-knockout mice as well as mice that overexpress SIRTI. In the present study, it was demonstrated that CIH decreased hepatic Sirt1 expression. RSV upregulated liver Sirt1 expression at the translational and transcriptional levels. It is postulated that activation of Sirt1 may be involved in the process by which RSV exerts its effect in rats, however, further investigation is required.

Insulin stimulates glucose uptake and its metabolism in the peripheral tissues. The insulin signaling pathway is fundamental in regulating blood insulin levels and maintaining glucose homeostasis. This pathway involves a series of signaling cascades that are activated by binding of insulin to its receptor, followed by autophosphorylation of the InsR, activation of the receptor tyrosine kinase and tyrosine phosphorylation of InsR substrates (IRSs). Phosphorylation of IRSs leads to activation of PI3K and subsequently to activation of serine/threonine kinase, AKT - a downstream mediator of PI3K signaling $(26,39)$. The PI3K/AKT pathway is a key component of the insulin signaling cascade and is considered necessary for glucose transport (38). Previous studies have demonstrated that insulin-stimulated PI3K activity decreases in the skeletal muscles of patients with type II diabetes $(40,41)$. In the current study, phosphorylation of PI3K and AKT decreased in the hepatic tissue following CIH exposure. RSV treatment significantly increased the fraction of phosphorylated PI3K and partially increased the fraction of phosphorylated AKT in the hepatic tissue. RSV also partially increased the total protein levels of PI3K and AKT in the hepatic tissue. These findings suggest that activation of the PI3K/AKT signaling pathway may contribute to the effects of RSV as demonstrated in the present study.

In conclusion, the current study has demonstrated that RSV effectively reverses IR in rats, which was induced by chronic hypoxia. Upregulation of Sirt1 and activation of PI3K/AKT signaling may be involved in this process. Without loss of function studies, it is difficult to form conclusions. Previous studies have reported that Sirt1 modulates insulin signaling by interacting with p85, the PI3K adapter subunit, in an insulin-independent manner (42). The direct interaction between Sirt1 and PI3K in regulating insulin signaling was not investigated in the present study. This is an important question, which is to be elucidated in future studies.

\section{Acknowledgements}

The authors would like to thank Medjaden Bioscience Limited for assisting in the preparation of this manuscript.

\section{References}

1. Punjabi NM. The epidemiology of adult obstructive sleep apnea. Proc Am Thorac Soc 5: 136-143, 2008.

2. Casale M, et al: Obstructive sleep apnea syndrome: from phenotype to genetic basis. Curr Genomics 10: 119-126, 2009.

3. Young T, Palta M, Dempsey J, Skatrud J, Weber S and Badr S: The occurrence of sleep-disordered breathing among middle-aged adults. N Engl J Med 328: 1230-1235, 1993.

4. Tasali E and Ip MS: Obstructive sleep apnea and metabolic syndrome: Alterations in glucose metabolism and inflammation. Proc Am Thorac Soc 5: 207-217, 2008.

5. Coughlin SR, Mawdsley L, Mugarza JA, Calverley PM and Wilding JP: Obstructive sleep apnoea is independently associated with an increased prevalence of metabolic syndrome. Eur Heart J 25: 735-741, 2004.

6. Ip MS, Lam B, Ng MM, Lam WK, Tsang KW and Lam KS: Obstructive sleep apnea is independently associated with insulin resistance. Am J Respir Crit Care Med 165: 670-676, 2002.

7. Punjabi NM, Shahar E, Redline S, Gottlieb DJ, Givelber R, Resnick HE and Sleep Heart Health Study I: Sleep-disordered breathing, glucose intolerance, and insulin resistance: The sleep heart health study. Am J Epidemiol 160: 521-530, 2004.

8. Goodson BL, Wung SF and Archbold KH: Obstructive sleep apnea hypopnea syndrome and metabolic syndrome: a synergistic cardiovascular risk factor. J Am Acad Nurse Pract 24: 695-703, 2012. 
9. Gao ZH, Yuan RY, Chen KY and Li GP: Obstructive sleep apnea and the metabolic syndrome. Sleep Breath 16: 937-938, 2012.

10. Byberg S, Hansen AL, Christensen DL, Vistisen D, Aadahl M, Linneberg A and Witte DR: Sleep duration and sleep quality are associated differently with alterations of glucose homeostasis. Diabet Med 29: e354-e360, 2012.

11. Van Cauter E: Sleep disturbances and insulin resistance. Diabet Med 28: 1455-1462, 2011.

12. Spiegel K, Knutson K, Leproult R, Tasali E and Van Cauter E: Sleep loss: A novel risk factor for insulin resistance and type 2 diabetes. J Appl Physiol 99: 2008-2019, 2005.

13. Finkel T, Deng CX and Mostoslavsky R: Recent progress in the biology and physiology of sirtuins. Nature 460: 587-591, 2009.

14. Chakraborty C and Doss CG: Sirtuins family-recent development as a drug target for aging, metabolism and age related diseases. Curr Drug Targets 14: 666-675, 2013.

15. Pucci B, Villanova L, Sansone L, Pellegrini L, Tafani M, Carpi A, Fini M and Russo MA: Sirtuins: The molecular basis of beneficial effects of physical activity. Intern Emerg Med 8 (Suppl 1): S23-S25, 2013.

16. Bagul PK and Banerjee SK: Insulin resistance, oxidative stress and cardiovascular complications: Role of sirtuins. Curr Pharm Des 19: 5663-5677, 2013

17. Chen YR, Lai YL, Lin SD, Li XT, Fu YC and Xu WC: Sirt1 interacts with metabolic transcriptional factors in the pancreas of insulin-resistant and calorie-restricted rats. Mol Biol Rep 40: 3373-3380, 2013

18. Jung TW, Lee KT, Lee MW and Ka KH: Sirtl attenuates palmitate-induced endoplasmic reticulum stress and insulin resistance in hepg2 cells via induction of oxygen-regulated protein 150. Biochem Biophys Res Commun 422: 229-232, 2012.

19. Wang RH, Kim HS, Xiao C, Xu X, Gavrilova O and Deng CX: Hepatic sirt1 deficiency in mice impairs mtorc2/akt signaling and results in hyperglycemia, oxidative damage and insulin resistance. J Clin Invest 121: 4477-4490, 2011.

20. Baur JA, Pearson KJ, Price NL, Jamieson HA, Lerin C, Kalra A, Prabhu VV, Allard JS, Lopez-Lluch G, Lewis K, Pistell PJ, Poosala S, Becker KG, Boss O, Gwinn D, Wang M, Ramaswamy S, Fishbein KW, Spencer RG, Lakatta EG, Le Couteur D, Shaw RJ, Navas P, Puigserver P, Ingram DK, de Cabo R and Sinclair DA: Resveratrol improves health and survival of mice on a high-calorie diet. Nature 444: 337-342, 2006.

21. Lagouge M, Argmann C, Gerhart-Hines Z, Meziane H, Lerin C, Daussin F, Messadeq N, Milne J, Lambert P, Elliott P, Geny B, Laakso M, Puigserver P and Auwerx J: Resveratrol improves mitochondrial function and protects against metabolic disease by activating sirtl and poc-1alpha. Cell 127: 1109-1122, 2006.

22. Tennen RI, Michishita-Kioi E and Chua KF: Finding a target for resveratrol. Cell 148: 387-389, 2012.

23. Gertz M, Nguyen GT, Fischer F, Suenkel B, Schlicker C, Franzel B, Tomaschewski J, Aladini F, Becker C, Wolters D and Steegborn C: A molecular mechanism for direct sirtuin activation by resveratrol. PloS One 7: e49761, 2012.

24. Iiyori N, Alonso LC, Li J, Sanders MH, Garcia-Ocana A, O'Doherty RM, Polotsky VY and O'Donnell CP: Intermittent hypoxia causes insulin resistance in lean mice independent of autonomic activity. Am J Respir Crit Care Med 175: 851-857, 2007.

25. Polotsky VY, Rubin AE, Balbir A, Dean T, Smith PL, Schwartz AR and O'Donnell CP: Intermittent hypoxia causes rem sleep deficits and decreases eeg delta power in nrem sleep in the c57bl/6j mouse. Sleep Med 7: 7-16, 2006.

26. Choi K and Kim YB: Molecular mechanism of insulin resistance in obesity and type 2 diabetes. Korean J Intern Med 25: 119-129, 2010.
27. Babacanoglu C, Yildirim N, Sadi G, Pektas MB and Akar F: Resveratrol prevents high-fructose corn syrup-induced vascular insulin resistance and dysfunction in rats. Food Chem Toxicol 60: 160-167, 2013.

28. Cheng AS, Cheng YH, Chiou $\mathrm{CH}$ and Chang TL: Resveratrol upregulates nrf2 expression to attenuate methylglyoxal-induced insulin resistance in hep g2 cells. J Agric Food Chem 60: 9180-9187, 2012.

29. Bagul PK, Middela H, Matapally S, Padiya R, Bastia T, Madhusudana K, Reddy BR, Chakravarty S and Banerjee SK: Attenuation of insulin resistance, metabolic syndrome and hepatic oxidative stress by resveratrol in fructose-fed rats. Pharmacol Res 66: 260-268, 2012.

30. Chen S, Li J, Zhang Z, Li W, Sun Y, Zhang Q, Feng X and Zhu W: Effects of resveratrol on the amelioration of insulin resistance in kkay mice. Can J Physiol Pharmacol 90: 237-242, 2012.

31. Zheng J, Chen LL, Zhang HH, Hu X, Kong W and Hu D: Resveratrol improves insulin resistance of catch-up growth by increasing mitochondrial complexes and antioxidant function in skeletal muscle. Metabolism 61: 954-965, 2012

32. Chen LL, Zhang HH, Zheng J, Hu X, Kong W, Hu D, Wang SX and Zhang P: Resveratrol attenuates high-fat diet-induced insulin resistance by influencing skeletal muscle lipid transport and subsarcolemmal mitochondrial beta-oxidation. Metabolism 60: 1598-1609, 2011

33. Labbé A, Garand C, Cogger VC, Paquet ER, Desbiens M, Le Couteur DG and Lebel M: Resveratrol improves insulin resistance hyperglycemia and hepatosteatosis but not hypertriglyceridemia, inflammation and life span in a mouse model for Werner syndrome. J Gerontol A Biol Sci Med Sci 66: 264-278, 2011.

34. Howitz KT, Bitterman KJ, Cohen HY, Lamming DW, Lavu S, Wood JG, Zipkin RE, Chung P, Kisielewski A, Zhang LL, Scherer B and Sinclair DA: Small molecule activators of sirtuins extend Saccharomyces cerevisiae lifespan. Nature 425: 191-196, 2003.

35. Yun JM, Chien A, Jialal I and Devaraj S: Resveratrol up-regulates sirtl and inhibits cellular oxidative stress in the diabetic milieu: Mechanistic insights. J Nutr Biochem 23: 699-705, 2012.

36. Liang F, Kume S and Koya D: Sirt1 and insulin resistance. Nat Rev Endocrinol 5: 367-373, 2009.

37. Zabolotny JM and Kim YB: Silencing insulin resistance through sirt1. Cell Metab 6: 247-249, 2007.

38. Farese RV, Sajan MP and Standaert ML: Insulin-sensitive protein kinases (atypical protein kinase $\mathrm{c}$ and protein kinase b/akt): Actions and defects in obesity and type II diabetes. Exp Biol Med (Maywood) 230: 593-605, 2005.

39. Cheatham B, Vlahos CJ, Cheatham L, Wang L, Blenis J and Kahn CR: Phosphatidylinositol 3-kinase activation is required for insulin stimulation of pp70 s6 kinase, DNA synthesis and glucose transporter translocation. Mol Cell Biol 14: 4902-4911, 1994.

40. Kim YB, Nikoulina SE, Ciaraldi TP, Henry RR and Kahn BB: Normal insulin-dependent activation of akt/protein kinase $b$, with diminished activation of phosphoinositide 3-kinase, in muscle in type 2 diabetes. J Clin Invest 104: 733-741, 1999.

41. Bjornholm M, Kawano Y, Lehtihet M and Zierath JR: Insulin receptor substrate-1 phosphorylation and phosphatidylinositol 3-kinase activity in skeletal muscle from niddm subjects after in vivo insulin stimulation. Diabetes 46: 524-527, 1997.

42. Frojdo S, Durand C, Molin L, Carey AL, El-Osta A, Kingwell BA, Febbraio MA, Solari F, Vidal H and Pirola L: Phosphoinositide 3-kinase as a novel functional target for the regulation of the insulin signaling pathway by sirt1. Mol Cell Endocrinol 335: 166-276, 2011. 